\title{
Estudio del Estado de Ánimo de las Mujeres Obesas de 30 a 45 Años Usuarias del Método Anticonceptivo Hormonal Trimestral en el Centro de Salud La Esperanza 2008
}

Responsable: $M g r$ : Rinna Pilco Velásquez

RESUMEN. El estudio permitió conocer el estado de ánimo de mujeres obesas que usan el método hormonal trimestral en el C.S. La Esperanza-Tacna. El diseño fue descriptiva transversal. Se trabajó con todas las mujeres usuarias del método hormonal trimestral con el diagnóstico de obesidad, siendo 42 según los criterios de inclusión y exclusión. Se aplicó una evaluación nutricional y un cuestionario de síntomas en salud mental (SRQ).Se llegaron a las siguientes conclusiones: Con respecto al estado de ánimo de las mujeres objeto de estudio, el $51 \%$ se siente nerviosa o tensa, revelando angustia, $28,6 \%$ se siente cansada todo el tiempo, lo cual revela un grado de depresión según el S.R.Q. aplicado. Asimismo se encontró que el $28,6 \%$ pasa por episodios de depresión. El IMCl en relación con el uso del método anticonceptivo revelo que el $14,3 \%$ de los caso pasaron de obesidad I a sobrepeso, lo cual indica que el inclremento de peso no se debe necesariamente al uso de MAC. Se recomienda estilos de vida saludable para contrarrestar el problema.
Miembro: Mgr. Olga Choque Chura

\begin{abstract}
The study allowed to know the state of mind of obese women who use the hormonal quarterly method in the C.S. The hope - Tacna. The design was a descriptive cross street. I work with all the women usuarias of hormonal quarterly method with the diagnosis of obesity, being 42 according to the criteria of incorporation and exclusion. I apply to him(you,them) a nutritional evaluation and a questionnaire of symptoms in mental Health (SRQ).Se they came to the following conclusions: With regard to the state of mind of the women I object of study, $51 \%$ feels nervous or tightens(tenses), revealing distress, $28,6 \%$ feels tired(tiring) all the time which reveals a degree of depression according to the applied(hardworking) S.R.Q.. Likewise one thought that 28,6\% happens(passes) for episodes of depression. The IMCl in relation to the use of the contraceptive Method I reveal that $14,3 \%$ of I them marry they went on from Obesity I to overweight, which indicates that the inclremento of weight does not owe necessarily to MAC's use. There are recommended ways of healthy life to offset the problem.
\end{abstract}

INTRODUCCIÓN. La defensa y promoción de los derechos de las mujeres, entre otros, el acceso a la planificación familiar, ha aumentado y diversificado sus oportunidades, permitiendo que las mujeres opten o adopten diversos estilos de vida, ya no limitándose a la maternidad y cuidado de los hijos, sino que ejecuten múltiples roles, integrándose al mundo laboral, académico, artístico, deportivo de alto rendimiento, etc.

El poder contar con un anticonceptivo hormonal oral que se adapte a sus estilos de vida, garantizándoles una alta efectividad anticonceptiva, ya no es considerada una opción, sino que es reclamado como un derecho por las mujeres actuales.

Cuando alguien se encuentra desanimado, se ve peor a sí mismo, y eso suele llevarle a un menor aprecio hacia sí mismo. Autoestima y estado de ánimo suelen ascender o descender de modo paralelo.

Una autoestima demasiado baja suele generar actitudes de frecuente desánimo, de no atreverse a casi nada, de desarrollar poco las propias capacidades y ver casi todo como inasequible. Con esa actitud, la derrota viene dada de antemano, antes de entrar en batalla, por esa injustificada infravaloración de uno mismo.

Cuando esa baja autoestima ha arraigado de modo profundo en una persona, hacerle comprender su error no será tarea fácil. Les cuesta mucho admitir cualquier valoración positiva de uno mismo, y cuando otras personas intentan hacérselo ver, con frecuencia lo interpreta como halagos infundados, simples cumplidos de cortesía, o bien como un ingenuo desconocimiento de la realidad, o incluso un intento de tomarles el pelo.

En la presente investigación se pretende colocar en evidencia cuál es el estado de ánimo de las mujeres de 30 a 45 años de edad que usan un método hormonal trimestral y que además han sido catalogadas con un estado nutricional de obesidad, hecho que repercute en la calidad de vida de la usuaria como mujer y su entorno familiar. 


\section{OBJETIVOS}

\section{Objetivo General}

Identificar el estado de ánimo de las mujeres obesas de 30 a 45 años usuarias del método anticonceptivo hormonal trimestral.

\section{Objetivos Específicos}

1. Establecer las características sociodemográficas de la población en estudio.

2. Determinar el tipo de obesidad en las usuarias del método hormonal trimestral.

3. Identificar el estado de salud mental (estado de ánimo).

\section{MATERIAL Y MÉTODO}

Se realizó un estudio descriptivo de corte transversal en el C.S. La Esperanza en el período comprendido entre mayo 2008 a abril 2009. Para efectos de la investigación se tomaron a todas las mujeres usuarias del método hormonal trimestral que tengan entre 30 a 45 años edad. Una vez identificadas se les realizó una entrevista individual en la que se recolectaron los datos necesarios y se aplicó el cuestionario de declaración de síntomas (Self-Reporting Questionnaire, SRQ) de la Organización Mundial de la Salud para conocer el estado de ánimo de la usuaria. Se precedió a registrar el peso anterior al uso del MAC y el peso actual. Además se aprovechó la oportunidad para dar consejería sobre nutrición y calidad de vida. Luego se realizó el análisis de cada uno de las variables mediante el uso del programa SPSS para tipificación de variables y aplicación de pruebas estadísticas, luego se procedió a realizar las contrastaciones de los resultados con la hipótesis y objetivos propuestos en el presente estudio.

\section{RESULTADOS}

Tabla 01: Distribución de frecuencias según caracteristicas sociodemográficas de las mujeres objeto de estudio. Tacna. 2008

\begin{tabular}{|c|c|c|c|c|c|c|}
\hline \multirow{2}{*}{\multicolumn{2}{|c|}{ CARACTERSTICA SOCIOOEMOCRAFICA }} & \multicolumn{5}{|c|}{ EOAO } \\
\hline & & \multirow{2}{*}{$\begin{array}{r}\text { De } 30 \text { a } \\
\text { 34 alos } \\
0\end{array}$} & \multirow{2}{*}{$\begin{array}{r}\begin{array}{c}\text { De } 36 \text { a } \\
39 \text { gias }\end{array} \\
4\end{array}$} & \multirow{2}{*}{$\begin{array}{r}\text { De } 40 \text { a } \\
44.0100 \\
1\end{array}$} & \multirow{2}{*}{$\frac{\text { Descaing }}{0}$} & \multirow{3}{*}{$\frac{1028}{5}$} \\
\hline \multirow{5}{*}{ EStADO ave } & Sodera & & & & & \\
\hline & & $.0 \%$ & $95 \%$ & & $.0 \%$ & \\
\hline & Cक्रada & 0 & 1 & 6 & 4 & 11 \\
\hline & & $.0 \%$ & $24 \%$ & $14,3 \%$ & $2.7 \%$ & $26.2 \%$ \\
\hline & Conviviente & $31, \% \%$ & $21,4 \%$ & $24 \%$ & $7, \% \frac{3}{4}$ & $61,9 \%$ \\
\hline \multirow[t]{4}{*}{ Thai } & & 13 & 14 & 8 & 7 & \\
\hline & & $37,0 \%$ & $30,3 \%$ & $90 \%$ & $16, \pi \%$ & $100 \% \%$ \\
\hline & Phimatia irrcomilata & 3 & 0 & 0 & 0 & 3 \\
\hline & & $\begin{array}{r}7, \% \\
2 \%\end{array}$ & $.0 \%$ & $.0 \%$ & $.0 \%$ & $7,1 \%$ \\
\hline \multirow{7}{*}{$\begin{array}{l}\text { NVEE DE } \\
\text { INSTRC COON }\end{array}$} & Primaia completa & $4 \%$ & $.0 \%$ & \begin{tabular}{r|r}
0 \\
$.0 \%$
\end{tabular} & $\begin{array}{r}0 \\
.0 \%\end{array}$ & $\begin{aligned} 2 \\
4 \% \%\end{aligned}$ \\
\hline & Secundria & 0 & 1 & 3 & 0 & 4 \\
\hline & ircamples & $0 \%$ & $2,4 \%$ & $7,7 \%$ & $0 \%$ & $9 \%$ \\
\hline & Secundria competa & $\begin{array}{r}8 \\
9,0 \%\end{array}$ & $\begin{array}{r}6 \\
143 \%\end{array}$ & $11,9 \%$ & $4 \%$ & $\begin{aligned} 2 \\
59.5 \%\end{aligned}$ \\
\hline & Superia técrico & 0 & & 0 & 1 & 2 \\
\hline & & $0 \%$ & $2.4 \%$ & $.0 \%$ & $2,4 \%$ & $48 \%$ \\
\hline & Speric uriverstatio & 0 & & 0 & 0 & 6 \\
\hline \multirow{2}{*}{\multicolumn{2}{|c|}{ Tadel }} & 13 & 14 & $\begin{array}{c}.0 \% \\
8\end{array}$ & $.0 \%$ & $\begin{aligned} 43 \% \\
4\end{aligned}$ \\
\hline & & max & $33,3 \%$ & $90 \%$ & $16, \pi \%$ & $1000 \%$ \\
\hline
\end{tabular}

Tabla 02: Distribución de frecuencia según tiempo de uso de MAC y edad de las mujeres objeto de estudio. Tacna.2008.

\begin{tabular}{|c|c|c|c|c|c|}
\hline \multirow{2}{*}{$\begin{array}{l}\text { TIEMPO DE USO OEL } \\
\text { MET ODO }\end{array}$} & \multicolumn{5}{|c|}{ EQAD } \\
\hline & $\begin{array}{l}\text { De } 303 \\
34 \text { arios } \\
\end{array}$ & $\begin{array}{l}\text { De } 35 \text { a } \\
39 \text { mias } \\
\end{array}$ & $\begin{array}{l}\text { De } 40 \text { a } \\
44 \operatorname{sins} \\
\end{array}$ & De 45 antos & Total \\
\hline Menos de 6 & 2 & 1 & 1 & 1 & 5 \\
\hline meses & $48 \%$ & $2.4 \%$ & $2,4 \%$ & $2.4 \%$ & $11,9 \%$ \\
\hline De 6 meses & 6 & 6 & 2 & 1 & 15 \\
\hline a 1 को & $14.3 \%$ & $14.3 \%$ & $4.8 \%$ & $2,4 \%$ & $35, \pi \%$ \\
\hline De 1 a 2 & 4 & 4 & 2 & 2 & 12 \\
\hline aŕos & $95 \%$ & $9.5 \%$ & $4.8 \%$ & $4.8 \%$ & $28.6 \%$ \\
\hline De 2 a 3 & 0 & 3 & 3 & 2 & 8 \\
\hline अवि s & $0 \%$ & $7.1 \%$ & $7,1 \%$ & $4.8 \%$ & $19,0 \%$ \\
\hline Mas de 3 & 1 & 0 & 0 & , & 2 \\
\hline ain 5 & $24 \%$ & $.0 \%$ & $.0 \%$ & $2,4 \%$ & $4.8 \%$ \\
\hline & 13 & 14 & $B$ & 7 & 42 \\
\hline Tord & 31.08 & $33.3 \%$ & $19,0 \%$ & $16.7 \%$ & $100.8 \%$ \\
\hline
\end{tabular}

Tabla 03: Distribución de frecuencias según el tiempo de uso del MAC y el indice de masa corporal (IMC) durante el uso del MAC en las mujeres objeto de estudio. Tacna. 2008.

\begin{tabular}{|c|c|c|c|c|}
\hline \multirow{2}{*}{$\begin{array}{l}\text { TIEMPO DE USO DEL } \\
\text { METODO }\end{array}$} & \multicolumn{4}{|c|}{ IMC. DURANTE USO DEL MAC } \\
\hline & Schrepeso & Ohesidad I & Obesidad : & Total \\
\hline \multirow{2}{*}{$\begin{array}{l}\text { Meros de } 6 \\
\text { meses }\end{array}$} & 2 & 3 & 0 & 5 \\
\hline & $4.8 \%$ & $7.1 \%$ & $.0 \%$ & $11.9 \%$ \\
\hline \multirow{2}{*}{$\begin{array}{l}\text { De } 6 \text { meses } \\
\text { a } 1 \text { ตั० }\end{array}$} & 4 & 9 & 2 & 15 \\
\hline & $9.5 \%$ & $21.4 \%$ & $4.8 \%$ & $35,7 \%$ \\
\hline \multirow{2}{*}{$\begin{array}{l}\text { De } 1 \text { a } 2 \\
\text { anos }\end{array}$} & 7 & 5 & 0 & 12 \\
\hline & $16.7 \%$ & $11.9 \%$ & $0 \%$ & $28.6 \%$ \\
\hline \multirow{2}{*}{$\begin{array}{l}\text { De } 2 \text { a } 3 \\
\text { años }\end{array}$} & 2 & 6 & 0 & 8 \\
\hline & $4,8 \%$ & $14.3 \%$ & $.0 \%$ & $19.0 \%$ \\
\hline \multirow{2}{*}{$\begin{array}{l}\text { Mas de } 3 \\
\text { años }\end{array}$} & 0 & 2 & 0 & 2 \\
\hline & $.0 \%$ & $4,8 \%$ & $.0 \%$ & $4,8 \%$ \\
\hline \multirow[t]{2}{*}{ Total } & 15 & 25 & 2 & 42 \\
\hline & $35.7 \%$ & $50.5 \%$ & $4.3 \%$ & $100.0 \%$ \\
\hline
\end{tabular}

Tabla 04: Distribución de frecuencia según el índice de Masa Corporal antes de usar el MAC en relación con el indice de Masa Corporal durante el uso del MAC de las mujeres objeto de estudio. Tacna.2008.

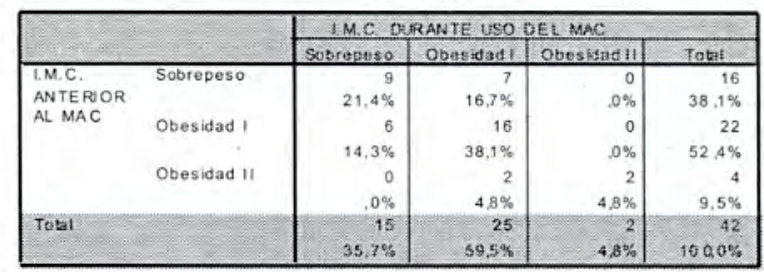

Tabla 05: Distribución de frecuencia según el índice de Masa Corporal y el nivel de instrucción y ocupación de las mujeres objeto de estudio. Tacna.2008.

\begin{tabular}{|c|c|c|c|c|c|}
\hline \multirow{2}{*}{\multicolumn{2}{|c|}{ FACTOR SOGODEMOGRARCO }} & \multicolumn{4}{|c|}{ I MC OURANIE USODELMAC } \\
\hline & & Sotzegenso & Obesidad 1 & Obesadad II & Totat \\
\hline \multirow[t]{10}{*}{$\begin{array}{l}\text { NIVEL DE } \\
\text { INSTRUCCICN }\end{array}$} & Primg la incomieta & $24{ }^{1}$ & $2{ }^{2}$ & $\begin{array}{r}0 \\
0 \%\end{array}$ & $\begin{array}{r}3 \\
771 \%\end{array}$ \\
\hline & Primria completa & 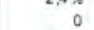 & 2 & 0 & 2 \\
\hline & & $.0 \%$ & $4.8 \%$ & $.0 \%$ & $4,8 \%$ \\
\hline & Secundria incompleta & 1 & 3 & 0 & 4 \\
\hline & & $2.4 \%$ & $7,1 \%$ & $.0 \%$ & $9,9 \%$ \\
\hline & Secundria campleta & & 15 & 1 & 25 \\
\hline & & $21,4 \%$ & $35.7 \%$ & $24 \%$ & $595 \%$ \\
\hline & Superia ténico & $24 \%$ & 1 & 0 & 2 \\
\hline & Superia uners trio & $\begin{array}{r}2.4 \% \\
3\end{array}$ & 2.476 & $0 \%$ & $4.8 \%$ \\
\hline & & $7.1 \%$ & $4.8 \%$ & $24 \%$ & $143 \%$ \\
\hline \multirow[t]{2}{*}{ Tata } & & 15 & 25 & 2 & 42 \\
\hline & & $35 \%$ & $595 \%$ & $4.8 \%$ & $100,0 \%$ \\
\hline \multirow[t]{8}{*}{ OCUPACION } & Estudarte & 0 & 1 & 0 & 1 \\
\hline & Comreinte & $\begin{aligned} 0 \% \\
2\end{aligned}$ & $2.4 \%$ & $.0 \%$ & $2,4 \%$ \\
\hline & Wortectane & $4.8 \%$ & $119 \%$ & $\begin{array}{r}0 \\
.0 \%\end{array}$ & $167 \%$ \\
\hline & Amadecasa & 10 & 16 & 1 & 27 \\
\hline & & $238 \%$ & $381 \%$ & $24 \%$ & $64,3 \%$ \\
\hline & Emplesde deperd ente & 3 & 1 & 1 & \\
\hline & & $7,1 \%$ & $2,4 \%$ & $2.4 \%$ & $11,9 \%$ \\
\hline & Indeperd ate & $\stackrel{0}{0 \%}$ & 2 & 0 & 2 \\
\hline \multirow{2}{*}{\multicolumn{2}{|c|}{ Tad }} & 15 & 25 & 2 & 42 \\
\hline & & $35 \%$ & $595 \%$ & $4.8 \%$ & $102 \pi$ \\
\hline
\end{tabular}


Tabla 06: Distribución de frecuencia según el Índice de Masa Corporal y la presencia de aspectos relacionados con la Angustia en las mujeres objeto de estudio. Tacna. 2008

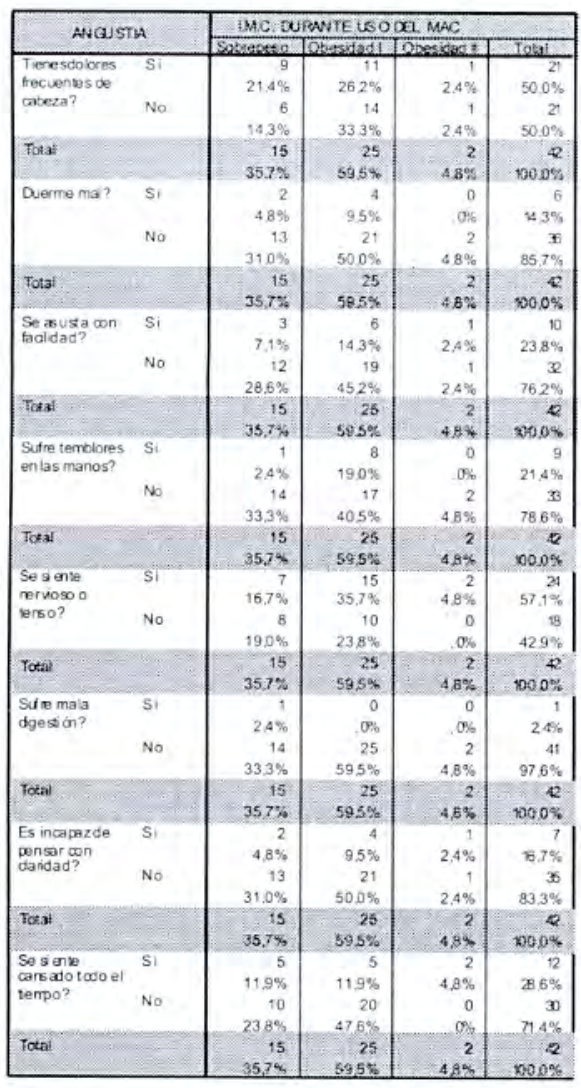

Tabla 07: Distribución de frecuencia según el Índice de Masa Corporal y la presencia de aspectos relacionados con la Depresión en las mujeres objeto de estudio. Tacna. 2008

\begin{tabular}{|c|c|c|c|c|c|}
\hline \multirow{2}{*}{\multicolumn{2}{|c|}{ 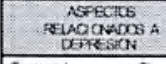 }} & \multicolumn{3}{|c|}{$\begin{array}{l}\text { NOEE DE MSA OCGPOAN } \\
\text { OURNIE LSODEL MAC }\end{array}$} & \multirow[b]{2}{*}{ Keal } \\
\hline & & Sorwoxes & anridad: & Oantiand & \\
\hline \multirow{4}{*}{$\begin{array}{l}\text { Tere ma! } \\
\text { apetio? }\end{array}$} & $s$ & 2 & 4 & 0 & 6 \\
\hline & & $48 \%$ & $9.7 \%$ & $.0 \%$ & $143 \%$ \\
\hline & No & 13 & 21 & $2^{2}$ & $\begin{array}{r}36 \\
857 \%\end{array}$ \\
\hline & & is & $500 \%$ & $485 \%$ & $857 \%$ \\
\hline tot & & 3578 & $59.5 \%$ & $43 \%$ & $1000 \%$ \\
\hline \multirow[t]{4}{*}{ Dusne mind } & s & 2 & 4 & 0 & \\
\hline & & $48 \%$ & $9.7 \%$ & $.0 \%$ & $14,3 \%$ \\
\hline & No & 13 & 21 & 2 & 36 \\
\hline & & $310 \%$ & $500 \%$ & 4886 & $857 \%$ \\
\hline \multirow{2}{*}{\multicolumn{2}{|c|}{ fota }} & 15 & 25 & 2 & $A_{2}$ \\
\hline \multirow{4}{*}{ Se senteteste? } & & 3578 & $595 \%$ & $48 \pi$ & $100, x$ \\
\hline & st & 2 & 2 & 1 & \\
\hline & & $48 \mathrm{BS}$ & $48 \%$ & 2416 & $11,9 \%$ \\
\hline & 78 & $310 \%$ & $548 \%$ & 246 & $881 \%$ \\
\hline \multirow[t]{2}{*}{ bon } & & 13 & 25 & 2 & 42 \\
\hline & & $357 \%$ & $595 \%$ & 406 & $100,0 \%$ \\
\hline $\begin{array}{l}\text { Ud lioracon } \\
\text { muha }\end{array}$ & s & 5 & 6 & 1 & 12 \\
\hline \multirow{2}{*}{$\begin{array}{l}\text { mucha } \\
\text { towencia? }\end{array}$} & $\mathrm{No}$ & $119 \%$ & $143 \%$ & $24 \%$ & $286 \%$ \\
\hline & & $238 \%$ & $\begin{array}{r}19 \\
4529\end{array}$ & & 30 \\
\hline \multirow[t]{2}{*}{ tots } & & 15 & 25 & $\begin{array}{r}246 \\
2\end{array}$ & $\begin{array}{l}714 \% \\
42\end{array}$ \\
\hline & & $357 \%$ & 5954 & $4 \pi x$ & 100,054 \\
\hline \multirow{4}{*}{$\begin{array}{l}\text { Hapersido } \\
\text { interis on las } \\
\text { coser? }\end{array}$} & $s$ & & & & \\
\hline & & $0 \%$ & $2,4 \%$ & $0 \%$ & $2.4 \%$ \\
\hline & To & 15 & 24 & $2^{2}$ & 41 \\
\hline & & $35 \%$ & $57,1 \%$ & $48 \%$ & $97.6 \%$ \\
\hline \multirow{2}{*}{ Fota } & & 15 & 25 & 2 & 42 \\
\hline & $\$$ & 3578 & 5958 & $4 \%$ & too, ans \\
\hline \multirow{3}{*}{$\begin{array}{l}\text { Se sentw } \\
\text { auride? }\end{array}$} & & $48 \times$ & $4,8 \%$ & $.0 \%$ & $99 \%$ \\
\hline & No & 13 & 23 & 2 & 38 \\
\hline & & $31.0 \%$ & $548 \%$ & $48 \%$ & $905 \%$ \\
\hline \multirow[t]{2}{*}{ sti } & & 15 & 25 & 2 & 42 \\
\hline & & $357 \%$ & 595\% & $48 x=$ & 100,05 \\
\hline \multirow{4}{*}{ 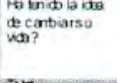 } & S & 4 & 6 & 1 & 11 \\
\hline & & $9.7 \%$ & $143 \%$ & $24 \%$ & $262 \%$ \\
\hline & No & 11 & 19 & 1 & 31 \\
\hline & & $26.2 \%$ & $452 \%$ & $24 \%$ & $738 \%$ \\
\hline \multirow{2}{*}{ sots } & & 15 & 25 & 2 & 42 \\
\hline & & $357 \%$ & $595 \%$ & $48 \%$ & 100,00 \\
\hline \multirow{3}{*}{$\begin{array}{l}\text { Se sonk } \\
\text { carsato bodod } \\
\text { temmo? }\end{array}$} & s & $119 \%$ & $119 \%$ & $4 \pi^{2}$ & $\begin{array}{r}12 \\
286 \%\end{array}$ \\
\hline & No & 10 & 20 & 0 & 30 \\
\hline & & $238 \%$ & $476 \%$ & $0 \%$ & $71,4 \%$ \\
\hline \multirow{2}{*}{\multicolumn{2}{|c|}{ का }} & 15 & 25 & 2 & 42 \\
\hline & & $327 \%$ & $595 \%$ & 4856 & $100,0 \%$ \\
\hline
\end{tabular}

Tabla 08: Distribución de frecuencia según el Índice de Masa Corporal y la presencia de aspectos relacionados con la Psicosis en las mujeres objeto de estudio. Tacna. 2008

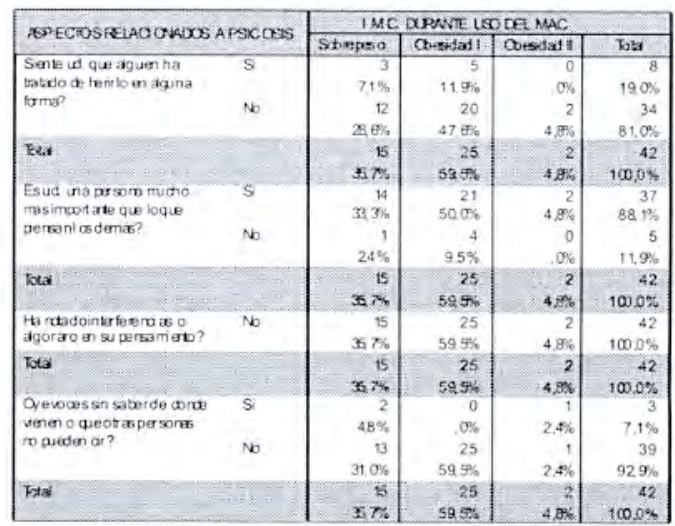

\section{DISCUSIÓN}

La obesidad está actualmente en los titulares de los periódicos. Y ello al menos por dos motivos. Primero, el número de personas obesas aumenta rápidamente en todo el mundo, hasta el punto de que la Organización Mundial de la Salud ha declarado que la obesidad es una epidemia mundial. Segundo, y la historia humana está detrás de los números, con la obesidad aumenta el riesgo de que la persona contraiga numerosas enfermedades debilitantes o posiblemente mortales. ¿Sabía Ud., por ejemplo, que según informes, la obesidad es la segunda de las causas de muertes evitables, después del hábito de fumar?

Según estimaciones, la obesidad afecta al $18 \%$ de la población mundial, cifra que representa un aumento de más del $50 \%$ en los siete últimos años. En todo el mundo hay unos 300 millones de adultos obesos y un número mucho mayor que padece exceso de peso.

Las mujeres objeto en estudio presentan ciertas características que hacen que sean predisponentes a la obesidad, como ser tener como ocupación ama de casa, lo cual conlleva a una rutina en la preparación de alimentos y esto podría contribuir al alto uso de carbohidratos, que en sus centros laborales consuman alimentos de expendio público que en su gran mayoría son frituras y potajes con rico contenido en carbohidratos reforzado con un sedentarismo nada favorable.

En este estudio no se ha observado que el uso de los MAC sean los causantes directos de obesidad, ya que algunas han modificado positivamente su indice de masa corporal durante el uso del método.

El cuestionario de declaración de síntomas (SelfReporting Questionnaire, SRQ) de la Organización Mundial de la Salud que se usó para conocer el estado de ánimo de la usuaria reveló que existe un grado de nerviosismo con una asociación a la presencia de dolores de cabeza, hay un nivel de depresión y autoestima baja, pero habría que profundizar más en los mismo para establecer las causas directas de los mismos. 
Los trabajos de investigación que se realicen en los sucesivo deberán tender a buscar las causas de obesidad de las mujeres usuarias de MAC y tomar un poco más atención en los estados de ánimo que tienen las mujeres, ya que este también esta dentro de los aspectos a cuidar dentro de la salud reproductiva.

\section{CONCLUSIONES}

1. Con respecto al estado de ánimo, un $57.1 \%$ se siente nerviosa o tensa, $50 \%$ presenta dolores de cabeza frecuente y un $28.6 \%$ refiere sentirse cansada todo el tiempo, lo cual revela un grado de angustia según el SRQ aplicado, el $28.6 \%$ de las mujeres en estudio manifiestan que lloran con mucha frecuencia y el $26.2 \%$ de ellas manifiestan que han tenido la idea de cambiar su vida lo cual esta relacionado a episodios de depresión según el SRQ.

2. El $88.1 \%$ revela sentirse una persona mucho mas importante de los que piensan los demás y un preocupante $7.1 \%$ revela oír voces que otras personas no pueden oir. Lo cual denota que la obesidad genera cambios en el estado de ánimo de las mujeres en estudio.

3. Con respecto al Índice de Masa Corporal (IMC) registrado durante el uso del MAC se observa que con Obesidad I se tiene que el $21.4 \%$ de ellas han utilizado el método de 6 meses a 1 año, seguido de las mujeres que lo usan de 2 a 3 años. Cabe destacar que el $59.5 \%$ de las mujeres en estudio durante el uso del MAC presentan Obesidad I y el $4.8 \%$ Obesidad II.

4. Al relacionarse el IMC anterior al uso del MAC con el IMC durante el uso del MAC, se encontró que $16.7 \%$ paso del sobre peso como diagnostico inicial a Obesidad I, otro grupo de mujeres en estudio pasaron de Obesidad I como diagnostico inicial pasaron a sobrepeso (14.3\%), así como 2 mujeres de Obesidad II pasaron a Obesidad I; hecho que evidencia que el incremento de peso no es necesariamente debido al uso del MAC.

5. La edad que predominó entre estas mujeres fue de 35 a 39 años $(33.3 \%)$, el $59,5 \%$ con Secundaria completa y como ocupación ama de casa en el $64,3 \%$ de los casos.

\section{RECOMENDACIONES}

Es un importante problema de salud, el cual conlleva repercusiones sanitarias negativas sobre las mujeres y, por ende, su entorno familiar y social. Se ha transmitido como una creencia el hecho de que una mujer que usa un método anticonceptivo tiene que subir de peso; sin embargo, se debe promover estilos de vida saludable que lleven a una salud física y mental como una alimentación balanceada, rica en vitaminas y fibras más que carbohidratos, habituar rutinas de ejercicios que estimulen el metabolismo, como ser caminatas, yoga, tai-chi, etc. paralelo a los cuidados de la salud reproductiva mediante el uso de anticonceptivos.

\section{REFERENCIAS BIBLIOGRÁFICAS}

BOWMAN, Bárbara A. y RUSSELL, Robert M. (2000) Conocimientos actuales sobre nutrición. Organización Panamericana de la Salud, Washington D.C. 20037, EUA, $8^{\circ}$ ed, pp. $441-452$.

Instituto Nacional de Salud, Centro Nacional de Alimentación y Nutrición- Dirección Ejecutiva de Vigilancia Alimentario Nutricional. (2005), Normalización de indicadores alimentarionutricionales. Lima.

MINISTERIO DE SALUD. (2004). Guias nacionales de atención integral de la salud sexual y reproductiva. Dirección General de Salud de las Personas, Minsa,Lima.

MINSA (2004). Lineamientos de nutrición materna. Resolución Ministerial Nro. 126-2004, 03 de febrero, p. 50 .

Monterrosa A: "Anticoncepción hormonal. EN : Caraballo J, Parra E, Taylor H. Memorias del $1^{\circ}$ Curso de Actualización en Ginecología y Pediatría. Imprenta U. de Cartagena. Cartagena. 1994; pp. 241 - 250.

Network en español. "Los métodos hormonales" y riesgos de contraer ITS". El asesoramiento siempre debe incluir los factores de riesgo de ITS. Revisado: Mayo 1512001 , Vol. 20, No. 4 En: http://www.fhi.org/sp/networks/sv20-4/ns2047.html. 